\title{
Article
}

\section{Common fixed point results of $s-\alpha$ contraction for a pair of maps in $b$-dislocated metric spaces}

\author{
Abdissa Fekadu ${ }^{1}$, Kidane Koyas ${ }^{1}$ and Solomon Gebregiorgis ${ }^{1, *}$ \\ 1 Department of Mathematics, Jimma University, Jimma, Ethiopia. \\ * Correspondence: solomonggty@gmail.com \\ Received: 9 September 2020; Accepted: 25 November 2020; Published: 16 December 2020.
}

\begin{abstract}
The purpose of this article is to construct fixed point theorems and prove the existence and uniqueness of common fixed point results of $s-\alpha$ contraction for a pair of maps in the setting of $b$ - dislocated metric spaces. Our results extend and generalize several well-known comparable results in the literature. The study procedure we used was that of Zoto and Kumari [1]. Furthermore, we provided an example in support of our main result.
\end{abstract}

Keywords: Fixed point, $s-\alpha$ contraction, $b$ - dislocated metric spaces.

MSC: 47H10, 54H25.

\section{Introduction}

$\mathbf{F}$ ixed point theory is an important tool in the study of nonlinear analysis as it is considered to be the key connection between pure and applied mathematics with wide applications in all branches of Mathematics, Economics, Biology, Chemistry, Physics and almost all engineering fields.

The famous Banach contraction principle is one of the powerful tools in metric fixed point theory and It has been extended and generalized in different directions by different researchers. The notion of metric space has been extended, improved and generalized in many different ways. Bakhtin [2] introduced a $b$ - metric space as a generalization of metric space and investigated some fixed point theorem in such spaces. Hitzler [3] introduced the notion of dislocated metric spaces. Zeyada et al., [4] generalized the results of Hitzler [3] and introduced the concept of complete dislocated quasi metric space. Aage et al., [5] proved common fixed point theorem in dislocated quasi $b$ - metric space. Zoto and Kumari [1] constructed theorems on common fixed point results on $b$ - dislocated metric spaces and proved the existence and uniqueness.

In this research work, we concentrate in establishing and proving common fixed point results for a pair of maps satisfying $s-\alpha$ contraction condition in the setting $b$ - dislocated metric spaces.

\section{Preliminaries}

Throughout this manuscript $\Re^{+}$represents the set of non-negative real numbers and $\mathbf{N}$ represents the set of natural numbers.

Definition 1. [6] Let $X$ be nonempty set and a mapping $d_{l}: X \times X \rightarrow \Re^{+}$is called a dislocated or $d_{l}$ - metric if the following conditions hold:

(a) $d_{l}(x, y)=0 \Rightarrow x=y$;

(b) $d_{l}(x, y)=d_{l}(y, x)$;

(c) $d_{l}(x, y) \leq d_{l}(x, z)+d_{l}(z, y)$, for all $x, y \in X$.

Then the pair $\left(X, d_{l}\right)$ is called a $d_{l}$ - metric space.

Definition 2. [7] Let $X$ be nonempty set and $s \geq 1$ be a real number, then a mapping $b_{d}: X \times X \rightarrow \Re^{+}$is called $b$ - dislocated metric if the following conditions hold: 
(a) $b_{d}(x, y)=0 \Rightarrow x=y$;

(b) $b_{d}(x, y)=b_{d}(y, x)$;

(c) $b_{d}(x, y) \leq s\left[b_{d}(x, z)+b_{d}(z, y)\right]$, for all $x, y, z \in X$.

Then the pair $\left(X, b_{d}\right)$ is called a $b$ - dislocated metric space.

Remark 1. The class of $b$ - dislocated metric space is larger than that of dislocated metric space.

Definition 3. Let $(X, d)$ be a metric space and $T: X \rightarrow X$ be a self-map, then $T$ is said to be a contraction map if there exists a constant $k \in[0,1)$ such that $d(T x, T y) \leq k d(x, y)$ for all $x, y \in X$.

Definition 4. [1] Let $\left(X, b_{d}\right)$ be a complete $b$ - dislocated metric space with parameter $s \geq 1$. If $T: X \rightarrow X$ is self-mapping that satisfy

$$
s^{2} b_{d}(T x, T y) \leq \alpha \max \left\{b_{d}(x, y), b_{d}(x, T x), b_{d}(y, T y), b_{d}(x, T y), b_{d}(y, T x)\right\}
$$

for all $x, y \in X$ and $\alpha \in\left[0, \frac{1}{2}\right)$, then $T$ is called a $s-\alpha$ quasi-contraction.

Lemma 1. Let $\left(X, b_{d}\right)$ be $a b$-dislocated metric space with parameter $s \geq 1$. Suppose that $\left\{x_{n}\right\}$ and $\left\{y_{n}\right\}$ are $b$ dislocated convergent to $x, y \in X$ respectively. Then we have

$$
\frac{1}{s^{2}} b_{d}(x, y) \leq \lim _{n \rightarrow \infty} \inf _{d}\left(x_{n}, y_{n}\right) \leq \lim _{n \rightarrow \infty} \operatorname{Sup}_{d}\left(x_{n}, y_{n}\right) \leq s^{2} b_{d}(x, y) .
$$

In particular, if $b_{d}\left(x_{n}, y_{n}\right)=0$, then we have $\lim _{n \rightarrow \infty} b_{d}\left(x_{n}, y_{n}\right)=0=b_{d}(x, y)$. Moreover, if each $z \in X$, we have

$$
\frac{1}{s} b_{d}(x, z) \leq \lim _{n \rightarrow \infty} \inf b_{d}\left(x_{n}, z\right) \leq \lim _{n \rightarrow \infty} \operatorname{Sup} b_{d}\left(x_{n}, z\right) \leq s b_{d}(x, z) .
$$

In particular, if $b_{d}(x, z)=0$, then we have $\lim _{n \rightarrow \infty} b_{d}\left(x_{n}, z\right)=0=b_{d}(x, z)$.

Theorem 1. [1] Let $\left(X, b_{d}\right)$ be complete $b$ - dislocated metric space with parameter $s \geq 1$. If $T: X \rightarrow X$ is a self-map that is a $s-\alpha$ quasi contraction, then $T$ has a unique fixed point in $X$.

Example 1. [1] Let $X=[0, \infty)$ and $b_{d}(x, y)=(x+y)^{2}$ for all $x, y \in X$. Then $b_{d}$ is a $b$-dislocated metric on $X$ with parameter $s=2$ and is complete.

Definition 5. [8] Let $\left(X, b_{d}\right)$ be a $b$ - dislocated metric space and $\left\{x_{n}\right\}$ be a sequence of points in $X$. A point $x \in X$ is said to be the limit of the sequence $\left\{x_{n}\right\}$ if $\lim _{n \rightarrow \infty} d\left(x_{n}, x\right)=0$ and we say that the sequence $\left\{x_{n}\right\}$ is $b$ dislocated convergent to $x$ and denote it by $x_{n} \rightarrow x$ as $n \rightarrow \infty$.

Lemma 2. [7] The limit of a convergent sequence in a $b$-dislocated metric space is unique.

Definition 6. [7] A sequence $\left\{x_{n}\right\}$ in a $b$ - dislocated metric space $\left(X, b_{d}\right)$ is called a $b$ - dislocated Cauchy sequence if and only if given $\epsilon>0$, there exists $n_{0} \in \mathbb{N}$ such that for all $n, m>n_{0}$, we have $b_{d}\left(x_{n}, x_{m}\right)<\epsilon$ or $\lim _{n, m \rightarrow \infty} d\left(x_{n} x_{m}\right)=0$.

Lemma 3. [7] Every $b$ - dislocated convergent sequence in $b$-dislocated metric space is $a b$-dislocated Cauchy.

Definition 7. [7] A $b$ - dislocated metric space $\left(X, b_{d}\right)$ is called complete if every $b$-dislocated Cauchy sequence in $X$ is a $b$ - dislocated convergent.

Definition 8. [6] Let $T: X \rightarrow X$ and $S: X \rightarrow X$ be self-maps in $\left(X, b_{d}\right)$. An element $x \in X$ is said to be a coincidence point of $T$ and $S$ if and only if $T x=S x=u$. A point $u \in X$ is point of coincidence of $T$ and $S$. 
Definition 9. [9] Let $T$ and $S$ be two self-maps on a metric space $(X, d)$. Then $T$ and $S$ are said to be weakly compatible if they commute at their coincident point; that is $T u=S u$ for some $u \in X$ implies $S T u=T S u$.

Definition 10. [10] Two self-maps $T: X \rightarrow X$ and $S: X \rightarrow X$ are said to be occasionally weakly compatible (OWC) if there exists some point $u \in X$ such that $T u=S u$ and $S T u=T S u$.

Remark 2. Clearly weakly compatible maps are occasionally weakly compatible. However the converse is not true in general.

Definition 11. [11] Let $T: X \rightarrow X$ and $S: X \rightarrow X$ be two self-maps on a metric space $(X, d)$. Then $T$ and $S$ are said to satisfy the common limit in the range of $S$ property, denoted by (CLRs) if there exists a sequence $\left\{x_{n}\right\} \in X$, such that

$$
\lim _{n \rightarrow \infty} T x_{n}=\lim _{n \rightarrow \infty} S x_{n}=S x
$$

for some $x \in X$.

Inspired and motivated by the result of Zoto and Kumari [1], the purpose of this research was to extend and generalize their main theorem to common fixed point theorem involving pairs of $s-\alpha$ contraction condition in the setting of $b$ - dislocated metric space.

\section{Main results}

In this section, we shall state and prove our main results.

Definition 12. Let $\left(X, b_{d}\right)$ be a $b$ - dislocated metric space with parameter $s \geq 1$. If $T, S: X \rightarrow X$ are self-mapping that satisfy

$$
s^{2} b_{d}(T x, T y) \leq \alpha \max \left\{b_{d}(S x, S y), b_{d}(S x, T x), b_{d}(S y, T y), b_{d}(S x, T y), b_{d}(S y, T x)\right\}
$$

for all $x, y \in X$ and $\alpha \in\left[0, \frac{1}{2}\right)$, then $T$ and $S$ are called an $s-\alpha$ contraction maps.

Theorem 2. Let $\left(X, b_{d}\right)$ be a complete $b$-dislocated metric space with parameter $s \geqslant 1$. If $T, S$ be self-maps of $X$ such that: The pair $(T, S)$ satisfy common limit in the range of $S$ property (CLRs) in $X$ and also $T$ and $S$ are $S-\alpha$ contraction maps, then

1. The pair $(T, S)$ has a coincidence point in $X$.

2. The pair $(T, S)$ has a unique common fixed point provided that $T$ and $S$ are weakly compatible mapping.

Proof. Since $T$ and $S$ satisfy (CLRs) property, there exists a sequence $\left\{x_{n}\right\} \in X$ such that:

$$
\lim _{n \rightarrow \infty} T x_{n}=\lim _{n \rightarrow \infty} S x_{n}=S u
$$

for some $u \in X$. By Equation (2), we have

$$
s^{2} b_{d}(T x, T y) \leq \alpha \max \left\{b_{d}(S x, S y), b_{d}(S x, T x), b_{d}(S y, T y), b_{d}(S x, T y), b_{d}(S y, T x)\right\} .
$$

By replacing $x=u$ and $y=x_{n}$ in the above condition, we obtain

$$
s^{2} b_{d}\left(T u, T x_{n}\right) \leq \alpha \max \left\{b_{d}\left(S u, S x_{n}\right), b_{d}(S u, T u), b_{d}\left(S x_{n}, T x_{n}\right), b_{d}(S u, T u), b_{d}\left(S x_{n}, T u\right)\right\} .
$$

By applying Lemma 1 and taking the upper limit as $n \rightarrow \infty$ on Equation (3), we get

$$
\begin{aligned}
s^{2} b_{d}(T u, S u) & \leq \alpha \max \left\{b_{d}(S u, S u), b_{d}(S u, T u), b_{d}(S u, S u), b_{d}(S u, S u), b_{d}(S u, T u)\right\} \\
& =\alpha \max \left\{b_{d}(S u, T u), b_{d}(S u, S u)\right\} .
\end{aligned}
$$


We consider the following cases as follows;

Case 1: If $\max \left\{b_{d}(S u, T u), b_{d}(S u, S u)\right\}=b_{d}(S u, T u)$, then we have $b_{d}(T u, S u) \leq \frac{\alpha}{s^{2}} b_{d}(S u, T u)$. It follows that $b_{d}(S u, T u)=0$ since $0 \leq \alpha<\frac{1}{2}$. Hence $S u=T u=z$ (say).

Case 2: If $\max \left\{b_{d}(S u, T u), b_{d}(S u, S u)\right\}=b_{d}(S u, S u)$, then we have

$$
\begin{aligned}
s^{2} b_{d}(T u, S u) & \leq \alpha b_{d}(S u, S u) \\
& \leq s \alpha\left[b_{d}(S u, T u)+b_{d}(T u, S u)\right] \\
& =2 s \alpha b_{d}(S u, T u) .
\end{aligned}
$$

It follows that $b_{d}(T u, S u) \leq \frac{2 \alpha}{s} b_{d}(S u, T u)$, which in turn implies that $b_{d}(S u, T u)=0$ since $0 \leq \alpha<\frac{1}{2}$. Hence $S u=T u=z$ (say). Therefore, $T$ and $S$ have a coincidence point. Now, by weakly compatibility property of the pair $(T, S)$, we have

$$
T z=T(S u)=S T u=S z
$$

which implies that $T z=S z$. Now, we show existence of a common fixed point. First, we show that $z$ is a fixed point of $T$. By Equation (2), we have

$$
s^{2} b_{d}\left(T z, T x_{n}\right) \leq \alpha \max \left\{b_{d}\left(S z, S x_{n}\right), b_{d}(S z, T z), b_{d}\left(S x_{n}, T x_{n}\right), b_{d}(S z, T z), b_{d}\left(S x_{n}, T z\right)\right\} .
$$

Taking the upper limit as $n \rightarrow \infty$, we get

$$
\begin{aligned}
s^{2} b_{d}(T z, z) & \leq \alpha \max \left\{\lim _{n \rightarrow \infty} \sup \left[b_{d}\left(S z, S x_{n}\right), b_{d}(S z, T z), b_{d}\left(S x_{n}, T x_{n}\right), b_{d}\left(S z, T x_{n}\right), b_{d}\left(S x_{n}, T z\right)\right]\right\} \\
& =\alpha \max \left\{b_{d}(S z, S u), b_{d}(S z, T z), b_{d}(S u, S u), b_{d}(S z, S u), b_{d}(S u, T z)\right\} .
\end{aligned}
$$

Since $S u=T u=z$ and $T z=S z$, we have

$$
\begin{aligned}
s^{2} b_{d}(T z, z) & \leq \alpha \max \left\{b_{d}(T z, z), b_{d}(S z, S z), b_{d}(z, z), b_{d}(T z, z), b_{d}(z, T z)\right\} \\
& =\alpha \max \left\{b_{d}(T z, z), b_{d}(T z, T z), b_{d}(z, z), b_{d}(T z, z), b_{d}(z, T z)\right\} .
\end{aligned}
$$

Now, we consider the following cases as follows;

Case 1: If $\max \left\{b_{d}(T z, z), b_{d}(T z, T z), b_{d}(z, z)\right\}=b_{d}(T z, z)$, then we have $b_{d}(T z, z) \leq \frac{\alpha}{s^{2}} b_{d}(T z, z)$, which implies $b_{d}(T z, z)=0$ since $0 \leq \alpha<\frac{1}{2}$. Hence it follows that $T z=z$.

Case 2: If $\max \left\{b_{d}(T z, z), b_{d}(T z, T z), b_{d}(z, z)\right\}=b_{d}(T z, T z)$, then we have

$$
\begin{aligned}
s^{2} b_{d}(T z, z) & \leq \alpha b_{d}(T z, T z) \\
& \leq s \alpha\left[b_{d}(z, T z)+b_{d}(T z, z)\right] \\
& =2 s \alpha b_{d}(T z, z) .
\end{aligned}
$$

It follows that $b_{d}(T z, z) \leq \frac{2 \alpha}{s} b_{d}(T z, z)$, which implies $b_{d}(T z, z)=0$ Since $0 \leq \alpha<\frac{1}{2}$. Hence $T z=z$. Case 3: If $\max \left\{b_{d}(T z, z), b_{d}(T z, T z), b_{d}(z, z)\right\}=b_{d}(z, z)$, then we have

$$
\begin{aligned}
s^{2} b_{d}(T z, z) & \leq \alpha b_{d}(z, z) \\
& \leq s \alpha\left[b_{d}(z, T z)+b_{d}(T z, z)\right] \\
& =2 s \alpha b_{d}(T z, z) .
\end{aligned}
$$

It follows that $b_{d}(T z, z) \leq \frac{2 \alpha}{s^{2}} b_{d}(T z, z)$, which implies $b_{d}(T z, z)=0$ since $0 \leq \alpha<\frac{1}{2}$. Hence $T z=z$. But we know that $T z=S z$ which gives us $T z=S z=z$. Therefore, $z$ is a common fixed point of $T$ and $S$.

\section{Uniqueness}

Let $z$ and $z$ l be fixed points of $T$ and $S$ with $z \neq z$. Then by Equation (2), we have

$$
\begin{aligned}
s^{2} b_{d}(T z, T z \prime) & \leq \alpha \max \left\{b_{d}(S z, S z \prime), b_{d}(S z, T z), b_{d}(S z \prime, T z \prime), b_{d}(S z, T z \prime), b_{d}(S z \prime, T z)\right\} \\
& =\alpha \max \left\{b_{d}(z, z \prime), b_{d}(z, z), b_{d}(z \prime, z \prime), b_{d}(z, z \prime), b_{d}(z \prime, z)\right\} .
\end{aligned}
$$


We consider the following cases as follows.

Case 1: If $\max \left\{b_{d}(z \prime, z), b_{d}(z, z), b_{d}(z \prime, z \prime)\right\}=b_{d}(z, z \prime)$, then we have $b_{d}(z \prime, z) \leq \frac{\alpha}{s^{2}} b_{d}(z, z \prime)$ which implies $b_{d}(z, z \prime)=0$ since $0 \leq \alpha<\frac{1}{2}$. Hence it follows that $z=z \prime$.

Case 2: If $\max \left\{b_{d}(z \prime, z), b_{d}(z, z), b_{d}(z \prime, z \prime)\right\}=b_{d}(z, z)$, then we have

$$
\begin{aligned}
s^{2} b_{d}\left(z, z^{\prime}\right) & \leq \alpha b_{d}(z, z) \\
& \leq s \alpha\left[b_{d}(z, z \prime)+b_{d}(z \prime, z)\right] \\
& =2 s \alpha b_{d}(z, z \prime) .
\end{aligned}
$$

It follows that $b_{d}(z, z \prime) \leq \frac{2 \alpha}{s} b_{d}(z, z \prime)$ which implies $b_{d}(z \prime, z)=0$ since $0 \leq \alpha<\frac{1}{2}$. Hence $z=z \prime$.

Case 3: If $\max \left\{b_{d}(z, z \prime), b_{d}(z, z), b_{d}(z \prime, z \prime)\right\}=b_{d}(z \prime, z \prime)$, then we have

$$
\begin{aligned}
s^{2} b_{d}(z, z \prime) & \leq \alpha b_{d}(z \prime, z \prime) \\
& \leq s \alpha\left[b_{d}(z, z \prime) b_{d}(z \prime, z)\right] \\
& =2 s \alpha b_{d}(z \prime, z) .
\end{aligned}
$$

It follows that $b_{d}(z \prime, z) \leq \frac{2 \alpha}{s} b_{d}(z \prime, z \prime)$ which implies $b_{d}(z \prime, z)=0$ since $0 \leq \alpha<\frac{1}{2}$. Hence $z \prime=z$ which contradicts to our assumption $z \neq z$.

Hence $z$ is a unique common fixed point of $T$ and $S$.

Theorem 3. Let $\left(X, b_{d}\right)$ be a complete $b$-dislocated metric space with parameter $s \geqslant 1$. If $T, S$ be self-maps of $X$ such that the pair $(T, S)$ satisfy occasionally weakly compatible property $(O W C)$ in $X$ and $T$ and $S$ are an $s-\alpha$ contraction maps. Then the pair $(T, S)$ has a unique common fixed point.

Proof. Since $T$ and $S$ satisfy (OWC) property, there exists a point $u \in X$ such that $T u=S u$ and $T S u=S T u$. This implies that $T S u=T T u=S T u=S S u$. It follows that $T T u=S S u$. We claim that $T u$ is the unique common fixed point of $T$ and $S$. First, we assert that $T u$ is a fixed point of $T$. For, if $T T u \neq T u$, then by Equation (2), we get

$$
s^{2} b_{d}(T u, T T u) \leq \alpha \max \left\{b_{d}(S u, S T u), b_{d}(S u, T u), b_{d}(S T u, T T u), b_{d}(S u, T T u), b_{d}(S T u, T u)\right\} .
$$

Since $S u=T u$, then we have

$$
\begin{aligned}
s^{2} b_{d}(T u, T T u) & \leq \alpha \max \left\{b_{d}(T u, T T u), b_{d}(T u, T u), b_{d}(T T u, T T u), b_{d}(T u, T T u), b_{d}(T T u, T u)\right\} \\
& =\alpha \max \left\{b_{d}(T u, T T u), b_{d}(T u, T u), b_{d}(T T u, T T u)\right\} .
\end{aligned}
$$

We consider the following three cases as follows.

Case 1: If $\max \left\{b_{d}(T u, T T u), b_{d}(T u, T u), b_{d}(T T u, T T u)\right\}=b_{d}(T u, T T u)$, then we have $b_{d}(T u, T T u) \leq$ $\frac{\alpha}{s^{2}} b_{d}(T u, T T u)$, which implies $b_{d}(T u, T T u)=0$ since $0 \leq \alpha<\frac{1}{2}$. Hence $T u=T T u$.

Case 2: If $\max \left\{b_{d}(T u, T T u), b_{d}(T u, T u), b_{d}(T T u, T T u)\right\}=b_{d}(T u, T u)$, then we have

$$
\begin{aligned}
s^{2} b_{d}(T u, T T u) & \leq \alpha b_{d}(T u, T u) \\
& \leq s \alpha\left[b_{d}(T T u, T u)+b_{d}(T u, T T u)\right] \\
& =2 s \alpha b_{d}(T T u, T u) .
\end{aligned}
$$

It follows that $b_{d}(T T u, T u) \leq \frac{2 \alpha}{s} b_{d}(T T u, T u)$, which implies that $b_{d}(T T u, T u)=0$ since $0 \leq \alpha<\frac{1}{2}$. Hence $T T u=T u$.

Case 3: If $\max \left\{b_{d}(T u, T T u), b_{d}(T u, T u), b_{d}(T T u, T T u)\right\}=b_{d}(T T u, T T u)$, then we have

$$
\begin{aligned}
s^{2} b_{d}(T u, T T u) & \leq \alpha b_{d}(T T u, T T u) \\
& \leq s \alpha\left[b_{d}(T T u, T u)+b_{d}(T u, T T u)\right] \\
& =2 s \alpha b_{d}(T T u, T u) .
\end{aligned}
$$


It follows that $b_{d}(T T u, T u) \leq \frac{2 \alpha}{s} b_{d}(T T u, T u)$, which implies that $b_{d}(T T u, T u)=0$ since $0 \leq \alpha<\frac{1}{2}$. Hence $T u=T T u$ which is a contradiction with $T u \neq T T u$. There fore, $T T u=T u$ and $T u$ is the fixed point of $T$. Since $T T u=T S u=S T u=T u=S S u$, it implies $S T u=T u$. Thus $T u$ is fixed point of $S$. Therefore, $T u$ is a common fixed point of $T$ and $S$.

\section{Uniqueness}

Suppose that $u, v \in X$ such that $T u=S u=u$ and $T v=S v=v$ and $u \neq v$. Then by Equation (2), we get

$$
\begin{aligned}
s^{2} b_{d}(u, v) & =s^{2} b_{d}(T u, T v) \\
& \leq \alpha \max \left\{b_{d}(S u, S v), b_{d}(S u, T u), b_{d}(S v, T v), b_{d}(S u, T v), b_{d}(S v, T u)\right\} \\
& =\alpha \max \left\{b_{d}(u, v), b_{d}(u, u), b_{d}(v, v), b_{d}(u, v), b_{d}(v, u)\right\} \\
& =\alpha \max \left\{b_{d}(u, v), b_{d}(u, u), b_{d}(v, v)\right\} .
\end{aligned}
$$

We consider the following cases as follows.

Case 1: If $\max \left\{b_{d}(u, v), b_{d}(u, u), b_{d}(v, v)\right\}=b_{d}(v, u)$, then we have $b_{d}(u, v) \leq \frac{\alpha}{s^{2}} b_{d}(v, u)$, which implies that $b_{d}(v, u)=0$, since $0 \leq \alpha<\frac{1}{2}$. Hence it follows that $u=v$.

Case 2: If $\max \left\{b_{d}(v, u), b_{d}(u, u), b_{d}(v, v)\right\}=b_{d}(u, u)$, then we have

$$
\begin{aligned}
s^{2} b_{d}(v, u) & \leq \alpha b_{d}(u, u) \\
& \leq s \alpha\left[b_{d}(u, v)+b_{d}(u, v)\right] \\
& =2 s \alpha b_{d}(u, v) .
\end{aligned}
$$

It follows that $b_{d}(v, u) \leq \frac{2 \alpha}{s} b_{d}(v, u)$, which implies that $b_{d}(v, u)=0$ since $0 \leq \alpha<\frac{1}{2}$. Hence $v=u$.

Case 3: If $\max \left\{b_{d}(u, v), b_{d}(v, v), b_{d}(u, u)\right\}=b_{d}(v, v)$, then we have

$$
\begin{aligned}
s^{2} b_{d}(u, v) & \leq \alpha b_{d}(v, v) \\
& \leq s \alpha\left[b_{d}(u, v) b_{d}(v, u)\right] \\
& =2 s \alpha b_{d}(u, v) .
\end{aligned}
$$

It follows that $b_{d}(u, v) \leq \frac{2 \alpha}{s} b_{d}(v, v)$, which implies that $b_{d}(v, u)=0$, since $0 \leq \alpha<\frac{1}{2}$. Hence $u=v$. Therefore, it contradicts with our assumption $u \neq v$.

Hence $u$ is a unique common fixed point of $T$ and $S$.

Theorem 4. Let $\left(X, b_{d}\right)$ be a complete $b$ - dislocated metric space with parameter $s \geqslant 1$. If $T, S$ be self-maps of $X$ such that

$$
s^{2} b_{d}(T x, T y) \leq \alpha \max \left\{b_{d}(S x, S y), b_{d}(S x, T x), b_{d}(S y, T y), b_{d}(S x, T y), b_{d}(S y, T x)\right\}
$$

for all $x, y \in X$ and $0 \leq \alpha<\frac{1}{2}$. Then the pair $(T, S)$ has a unique common fixed point.

Proof. Let $x_{0}$ be arbitrary given point in $X$. Define the sequence $y_{n} \in X$ such that; $y_{2 n}=T x_{2 n}=S x_{2 n+1}$, for all $n \geq 0$. We show that $y_{n} \in X$ for all $n \in \mathbb{N}$. Since $y_{2 n}=T x_{2 n}=S x_{2 n+1}$, we have from (1) that

$$
\begin{aligned}
s^{2} b_{d}\left(y_{2 n}, y_{2 n+1}\right)= & s^{2} b_{d}\left(T x_{2 n}, T x_{2 n+1}\right) \\
\leq & \alpha \max \left\{b_{d}\left(S x_{2 n}, S x_{2 n+1}\right), b_{d}\left(S x_{2 n}, T x_{2 n}\right), b_{d}\left(S x_{2 n+1}, T x_{2 n+1}\right), b_{d}\left(S x_{2 n}, T x_{2 n+1}\right)\right. \\
& \left.b_{d}\left(S x_{2 n+1}, T x_{2 n} x\right)\right\} \\
= & \alpha \max \left\{b_{d}\left(y_{2 n-1}, y_{2 n}\right), b_{d}\left(y_{2 n-1}, y_{2 n}\right), b_{d}\left(y_{2 n}, y_{2 n+1}\right), b_{d}\left(y_{2 n-1}, y_{2 n+1}\right), b_{d}\left(y_{2 n}, y_{2 n}\right)\right\} \\
\leq & \alpha \max \left\{b_{d}\left(y_{2 n-1}, y_{2 n}\right), b_{d}\left(y_{2 n-1}, y_{2 n}\right), b_{d}\left(y_{2 n}, y_{2 n+1}\right), s\left[b_{d}\left(y_{2 n-1}, y_{2 n}\right)+b_{d}\left(y_{2 n},\right.\right.\right. \\
& \left.\left.y_{2 n+1}\right)\right], s\left[b_{d}\left(y_{2 n}, y_{2 n+1}+b_{d}\left(y_{2 n-1}, y_{2 n}\right)\right]\right\}
\end{aligned}
$$




$$
\begin{aligned}
= & \alpha \max \left\{b_{d}\left(y_{2 n-1}, y_{2 n}\right), b_{d}\left(y_{2 n-1}, y_{2 n}\right), b_{d}\left(y_{2 n}, y_{2 n+1}\right), s\left[b_{d}\left(y_{2 n-1}, y_{2 n}\right)+b_{d}\left(y_{2 n},\right.\right.\right. \\
& \left.\left.\left.y_{2 n+1}\right)\right], 2 s\left[b_{d}\left(y_{2 n-1}, y_{2 n}\right)\right]\right\} .
\end{aligned}
$$

If $b_{d}\left(y_{2 n-1}, y_{2 n}\right) \leq b_{d}\left(y_{2 n}, y_{2 n+1}\right)$, for some $n \in \mathbb{N}$, then by (1), we have $s^{2} b_{d}\left(y_{2 n}, y_{2 n+1}\right) \leq 2 \alpha b_{d}\left(y_{2 n}, y_{2 n+1}\right)$, which implies that $b_{d}\left(y_{2 n}, y_{2 n+1}\right) \leq \frac{2 \alpha}{s^{2}} b_{d}\left(y_{2 n}, y_{2 n+1}\right)$. This is not true because $\frac{2 \alpha}{s^{2}}<1$. Thus $b_{d}\left(y_{2 n}, y_{2 n+1}\right) \leq$ $b_{d}\left(y_{2 n-1}, y_{2 n}\right)$ for all $n \in \mathbb{N}$. Also, by the above inequality, we get

$$
\begin{aligned}
s^{2} b_{d}\left(y_{2 n}, y_{2 n+1}\right) & \leq 2 \alpha s b_{d}\left(y_{2 n-1}, y_{2 n}\right), \\
b_{d}\left(y_{2 n-1}, y_{2 n}\right) & \leq \frac{2 \alpha}{s} b_{d}\left(y_{2 n-2}, y_{2 n-1}\right), \\
b_{d}\left(y_{2 n-2}, y_{2 n-1}\right) & \leq \frac{2 \alpha}{s} b_{d}\left(y_{2 n-3}, y_{2 n-4}\right) .
\end{aligned}
$$

Continuing like this, we have

$$
b_{d}\left(y_{2 n}, y_{2 n+1}\right) \leq c b_{d}\left(y_{2 n-1}, y_{2 n}\right) \leq c^{2} b_{d}\left(y_{2 n-2}, y_{2 n-1}\right), \cdots \leq c^{n} b_{d}\left(y_{0}, y_{1}\right)
$$

where $c=\frac{2 \alpha}{s}$ and $0 \leq c<1$. Taking the upper limit as $n \rightarrow \infty$ in the inequality above, we have $b_{d}\left(y_{2 n}, y_{2 n+1}\right) \rightarrow 0$. Now, we prove that $\left\{y_{m}\right\}$ is a $b$-dislocated Cauchy sequence where $m=2 n$. To do this let $m, n \geq 0$ with $m>n$. Now, using the triangle inequality, we have

$$
\begin{aligned}
b_{d}\left(y_{n}, y_{m}\right) & \leq s\left[b_{d}\left(y_{n}, y_{n+1}\right)+b_{d}\left(y_{n+1}, y_{m}\right)\right] \\
& \leq s\left[b_{d}\left(y_{n}, y_{n+1}\right)+s\left[b_{d}\left(y_{n+1}, y_{n+2}\right)+b_{d}\left(y_{n+2}, y_{m}\right)\right]\right] \\
& \leq s b_{d}\left(y_{n}, y_{n+1}\right)+s^{2} b_{d}\left(y_{n+1}, y_{n+2}\right)+\cdots+s^{n} b_{d}\left(y_{m-1}, y_{m}\right) \\
& =s\left[c^{n} b_{d}\left(y_{0}, y_{1}\right)+s c^{n+1} b_{d}\left(y_{0}, y_{1}\right)+\cdots\right] \\
& =s c^{n}\left[b_{d}\left(y_{0}, y_{1}\right)+s c b_{d}\left(y_{0}, y_{1}\right)+\cdots\right] \\
& =s c^{n} b_{d}\left(y_{0}, y_{1}\right)\left[1+s c+(s c)^{2}+\cdots\right] \\
& \leq \frac{s c^{n}}{(1-s c)} b_{d}\left(y_{0}, y_{1}\right) .
\end{aligned}
$$

Therefore $b_{d}\left(y_{n}, y_{m}\right) \leq \frac{s c^{n}}{(1-s c)} b_{d}\left(y_{0}, y_{1}\right)$. Taking the upper limit as $m, n \rightarrow \infty$, we have $b_{d}\left(y_{n}, y_{m}\right) \rightarrow 0$ as $s c<1$. Therefore, $\left\{y_{m}\right\}$ is a $b$-dislocated Cauchy sequence in $b$-dislocated metric space $\left(X, b_{d}\right)$. So there is some $u \in X$ such that $\left\{y_{m}\right\}$ is a $b$-dislocated converges to $u$. Since a subsequence of a Cauchy sequence in $b$ -dislocated metric space is a Cauchy sequence, then $\left\{T x_{2 n}\right\}$ and $\left\{S x_{2 n+1}\right\}$ are also Cauchy sequences.

If $T$ and $S$ are continuous mappings, we get

$$
T(u)=T\left(\lim _{n \rightarrow \infty} x_{n}\right)=\lim _{n \rightarrow \infty} T x_{n}=S(u)=\lim _{n \rightarrow \infty} S x_{n+1}=u .
$$

Thus, $u$ is a common fixed point of $T$ and $S$. If the self-map $T$ is not continuous then, we consider

$$
\begin{aligned}
s^{2} b_{d}\left(y_{2 n}, T u\right) & =s^{2} b_{d}\left(T x_{2 n}, T u\right) \\
& \leq \alpha \max \left\{b_{d}\left(S x_{2 n}, S u\right), b_{d}\left(S x_{2 n}, T x_{2 n}\right), b_{d}(S u, T u), b_{d}\left(S x_{2 n}, T u\right), b_{d}\left(S u, T x_{2 n}\right)\right\} \\
& =\alpha \max \left\{b_{d}\left(y_{2 n-1}, S u\right), b_{d}\left(y_{2 n-1}, y_{2 n}\right), b_{d}(S u, T u), b_{d}\left(y_{2 n-1}, T u\right), b_{d}\left(S u, y_{2 n}\right)\right\} .
\end{aligned}
$$

On taking upper limit as $n \rightarrow \infty$, we get $s^{2} b_{d}(u, T u) \leq \alpha \max \left\{b_{d}(S u, S u), b_{d}(S u, T u), b_{d}(S u, T u)\right\}$. We consider the following cases:

Case 1: If $\max \left\{b_{d}(u, u), b_{d}(T u, T u), b_{d}(u, T u)\right\}=b_{d}(T u, u)$, then we have $b_{d}(T u, u) \leq \frac{\alpha}{s^{2}} b_{d}(T u, u)$, which 
implies $b_{d}(T u, u)=0$, since $0 \leq \alpha<\frac{1}{2}$. Hence $T u=u$.

Case 2: If $\max \left\{b_{d}(u, u), b_{d}(T u, T u), b_{d}(T u, u)\right\}=b_{d}(T u, T u)$, then we have

$$
\begin{aligned}
s^{2} b_{d}(T u, u) & \leq \alpha b_{d}(T u, T u) \\
& \leq s \alpha\left[b_{d}(u, T u)+b_{d}(T u, u)\right] \\
& =2 s \alpha b_{d}(T u, u) .
\end{aligned}
$$

It follows that $b_{d}(T u, u) \leq \frac{2 \alpha}{s} b_{d}(T u, u)$, which implies $b_{d}(T u, u)=0$ since $0 \leq \alpha<\frac{1}{2}$. Hence $T u=u$.

Case 3: If $\max \left\{b_{d}(u, u), b_{d}(T u, T u), b_{d}(T u, u)\right\}=b_{d}(u, u)$, then we have

$$
\begin{aligned}
s^{2} b_{d}(T u, u) & \leq \alpha b_{d}(u, u) \\
& \leq s \alpha\left[b_{d}(u, T u) b_{d}(T u, u)\right] \\
& =2 s \alpha b_{d}(T u, u) .
\end{aligned}
$$

It follows that $b_{d}(T u, u) \leq \frac{2 \alpha}{s} b_{d}(T u, u)$, which implies $b_{d}(T u, u)=0$ since $0 \leq \alpha<\frac{1}{2}$. Hence $T u=u$.

In all cases $b_{d}(u, T u)=0$ which implies that $u=T u$. Thus, $u$ is fixed point of $T$.

In similar cases, we have $b_{d}(S u, u) \leq \frac{2 \alpha}{s} b_{d}(S u, u)$, which implies $b_{d}(S u, u)=0$ since $0 \leq \alpha<\frac{1}{2}$. Hence $S u=u$. Thus, $u$ is fixed point of $S$. Since, $S u=u=T u$, then $u$ is a common fixed point of $T$ and $S$.

\section{Uniqueness}

Let $u$ and $v$ are fixed points of $T$ and $S$ with $u \neq v$. Then by using Equation (2), we have

$$
\begin{aligned}
s^{2} b_{d}(u, v)=s^{2} b_{d}(T u, T v) \leq & \alpha \max \left\{b_{d}(S u, S v), b_{d}(S u, T u), b_{d}(S v, T v), b_{d}(S u, T v), b_{d}(S v, T u)\right\} \\
& =\alpha \max \left\{b_{d}(u, v), b_{d}(u, u), b_{d}(v, v), b_{d}(u, v), b_{d}(v, u)\right\} \\
& =\alpha \max \left\{b_{d}(u, v), b_{d}(v, v), b_{d}(v, u)\right\} .
\end{aligned}
$$

We consider the following three cases;

Case 1: If $\max \left\{b_{d}(u, v), b_{d}(u, u), b_{d}(v, v)\right\}=b_{d}(v, u)$, then we have $b_{d}(u, v) \leq \frac{\alpha}{s^{2}} b_{d}(v, u)$, which implies $b_{d}(v, u)=0$ since $0 \leq \alpha<\frac{1}{2}$. Hence it follows that $u=v$.

Case 2: If $\max \left\{b_{d}(v, u), b_{d}(u, u), b_{d}(v, v)\right\}=b_{d}(u, u)$, then we have

$$
\begin{aligned}
s^{2} b_{d}(v, u) & \leq \alpha b_{d}(u, u) \\
& \leq s \alpha\left[b_{d}(u, v)+b_{d}(u, v)\right] \\
& =2 s \alpha b_{d}(u, v) .
\end{aligned}
$$

It follows that $b_{d}(v, u) \leq \frac{2 \alpha}{s} b_{d}(v, u)$, which implies $b_{d}(v, u)=0$ since $0 \leq \alpha<\frac{1}{2}$. Hence it follows that $v=u$. Case 3: If $\max \left\{b_{d}(u, v), b_{d}(v, v), b_{d}(u, u)\right\}=b_{d}(v, v)$, then we have

$$
\begin{aligned}
s^{2} b_{d}(v, u) & \leq \alpha b_{d}(v, v) \\
& \leq s \alpha\left[b_{d}(u, v)+b_{d}(v, u)\right] \\
& =2 s \alpha b_{d}(u, v) .
\end{aligned}
$$

It follows that $b_{d}(u, v) \leq \frac{2 \alpha}{s} b_{d}(v, u)$, which implies $b_{d}(v, u)=0$ since $0 \leq \alpha<\frac{1}{2}$. Hence in all cases $u=v$. Therefore, it contradicts with our assumption $u \neq v$. Hence $u$ is a unique common fixed point of $T$ and $S$.

Remark 3. If we take $S=I \quad(I$ is the identity map on $X)$ in Theorem 4 , we get Theorem 1 .

Now, we give an example in support of Theorem 4.

Example 2. Let $X=[0,1]$ and $b_{d}(x, y)=(x+y)^{2}$ for all $x, y \in X$ when $s=2$ is a b-dislocated metric on $X$. Then $\left(X, b_{d}\right)$ is a b-dislocated metric space. 
Solution. We take the $s-\alpha$ contraction map and define the following

$$
T x=\left\{\begin{array}{l}
\frac{1}{25} x, \text { if } x \in[0,1) \\
\frac{1}{30}, \text { if } x=1
\end{array} \quad \text { and } \quad S x=\frac{1}{5} x .\right.
$$

Consider the sequence $\left\{x_{n}\right\}$ given by $X_{n}=\frac{1}{n}$ for all $n \in \mathbf{N}$. T and $S$ satisfies the common limit in the range of $S$ property. By using (CLRs) properties, we have $\lim _{n \rightarrow \infty} T x_{n}=\lim _{n \rightarrow \infty} S x_{n}=0$, for $0 \in T x$ or $0 \in S x$. We have the following cases with $\alpha=\frac{4}{25}$.

Case 1: For $x, y \in[0,1)$, we have

$$
s^{2} b_{d}(T x, T y)=4 b_{d}\left(\frac{1}{25} x, \frac{1}{25} y\right)=4\left(\frac{1}{25} x+\frac{1}{25} y\right)^{2}=\frac{4}{25}\left(\frac{1}{5} x+\frac{1}{5} y\right)^{2} \leq \frac{4}{25} b_{d}(S x, S y) .
$$

Therefore, $s^{2} b_{d}(T x, T y) \leq \alpha b_{d}(S x, S y)$.

Case 2: For $y<x$ and $x=1$, we have

$$
s^{2} b_{d}(T 1, T y)=4 b_{d}\left(\frac{1}{30}, \frac{1}{25} y\right)=4\left(\frac{1}{30}+\frac{1}{25} y\right)^{2} \leq \frac{4}{25}\left(\frac{1}{5}+\frac{1}{5} y\right)^{2}=\frac{4}{25} b_{d}\left(\frac{1}{5}, \frac{1}{5} y\right) \leq \frac{4}{25} b_{d}(S x, S y) .
$$

Therefore, $s^{2} b_{d}(T 1, T y) \leq \alpha b_{d}(S 1, S y)=\alpha b_{d}(S x, S y)$.

Case 3: For $x<y$ and $y=1$, we have

$$
s^{2} b_{d}(T x, T 1)=4 b_{d}\left(\frac{1}{25} x, \frac{1}{30}\right)=4\left(\frac{1}{25} x+\frac{1}{30}\right)^{2} \leq \frac{4}{25}\left(\frac{1}{5} x+\frac{1}{5}\right)^{2}=\frac{4}{25} b_{d}\left(\frac{1}{5} x, \frac{1}{5}\right) \leq \frac{4}{25} b_{d}(S x, S y)
$$

Therefore, $s^{2} b_{d}(T x, T 1) \leq \alpha b_{d}(S x, S 1)=\alpha b_{d}(S x, S y)$.

Case 4: For $x=y=1$, we have

$s^{2} b_{d}(T 1, T 1)=4 b_{d}\left(\frac{1}{30}, \frac{1}{30}\right)=\frac{4}{25}\left(\frac{1}{6}+\frac{1}{6}\right)^{2} \leq \frac{4}{25}\left(\frac{1}{5}+\frac{1}{5}\right)^{2}=\frac{4}{25} b_{d}\left(\frac{1}{5}, \frac{1}{5}\right)=\frac{4}{25} b_{d}(S 1, S 1) \leq \frac{4}{25} b_{d}(S x, S y)$.

Therefore, $s^{2} b_{d}(T 1, T 1) \leq \alpha b_{d}(S 1, S 1)=\alpha b_{d}(S x, S y)$.

From cases 1 up to 4 , we see that all the conditions of Theorem 4 are satisfied and 0 is the unique common fixed point of $T$ and $S$.

\section{Conclusion}

Zoto and Kumari [1] established the existence and uniqueness of fixed point for a mapping satisfying $s-\alpha$ type contraction condition in a complete dislocated metric space. In this thesis, we have explored the properties of $s-\alpha$ type contraction mapping in $b$ - dislocated metric spaces. We established the theorem on common fixed points of two mapping satisfying $s-\alpha$ contraction condition in the setting of $b$ - dislocated metric spaces and proved the existence and uniqueness of common fixed point for a pair of maps $\mathrm{T}$ and $\mathrm{S}$ in the setting of $b$ - dislocated metric space. Also we provided an example in support of our main results. Our work extended fixed point result in single map to common fixed point result in a pair of maps. The presented theorem extends and generalizes several well-known comparable results in literature.

Acknowledgments: The authors would like to thank the College of Natural Sciences, Jimma University for funding this research work.

Author Contributions: All authors contributed equally to the writing of this paper. All authors read and approved the final manuscript.

Conflicts of Interest: "The authors declare no conflict of interest."

\section{References}


[1] Zoto, K., \& Kumari, P. S. (2019). Fixed point theorems for $s-\alpha$ contractions in dislocated and $b$-dislocated metric space. Thai Journal of Mathematics, 17(1), 263-276

[2] Bakhtin, I. (1989). The contraction mapping principle in quasimetric spaces. Functional Analysis, Gos. Ped. Inst. Unianowsk, 30, 26-37.

[3] Hitzler, P., \& Seda, A. K. (2000). Dislocated topologies. Journal of Electrical Engineering, 51(12), 3-7.

[4] Zeyada, F. M., Hassan, G. H., \& Ahmed, M. A. (2006). A generalization of a fixed point theorem due to Hitzler and Seda in dislocated quasi-metric spaces. Arabian journal for Science and Engineering, 31(1A), 111.

[5] Aage, C. T., \& Golhare, P. G. (2016). On fixed point theorems in dislocated quasi $b$-metric spaces. International Journal of Advances in Matematics, 2016 (1), 55-70.

[6] Sintunavarat, W., \& Kumam, P. (2011). Common fixed point theorems for a pair of weakly compatible mappings in fuzzy metric spaces. Journal of Applied mathematics, 2011, Article ID 637958.

[7] Kutbi, M. A., Arshad, M., Ahmad, J., \& Azam, A. (2014). Generalized common fixed point results with applications. Abstract and Applied Analysis, 2014, Article ID 363925.

[8] Hussain, N., Roshan, J. R., Parvaneh, V., \& Abbas, M. (2013). Common fixed point results for weak contractive mappings in ordered $b$-dislocated metric spaces with applications. Journal of Inequalities and Applications, 2013, Article No. 486.

[9] Jungck, G. (1996). Common fixed points for noncontinuous nonself maps on nonmetric spaces. Far East Journal of Mathematical Sciences, 4(2), 199-215.

[10] Jungck, G., \& Rhoades, B. E. (2006). Fixed point theorems for occasionally weakly compatible mappings. Fixed Point Theory, 7(2), 287-296.

[11] Verma, R. K., \& Pathak, H. K. (2012). Common fixed point theorems using property (EA) in complex-valued metric spaces. Thai Journal of Mathematics, 11(2), 347-355.

[12] Banach, S. (1922). Sur les opérations dans les ensembles abstraits et leur application aux équations intégrales. Fundamenta Mathematicae, 3(1), 133-181.

(C) 2020 by the authors; licensee PSRP, Lahore, Pakistan. This article is an open access article distributed under the terms and conditions of the Creative Commons Attribution (CC-BY) license (http://creativecommons.org/licenses/by/4.0/). 